\title{
Molnupiravir: coding for catastrophe
}

\author{
Molnupiravir, a wide-spectrum antiviral that is currently in phase 2/3 clinical trials for the treatment of COVID-19, \\ is proposed to inhibit viral replication by a mechanism known as 'lethal mutagenesis'. Two recently published \\ studies reveal the biochemical and structural bases of how molnupiravir disrupts the fidelity of SARS-CoV-2 \\ genome replication and prevents viral propagation by fostering error accumulation in a process referred to as \\ 'error catastrophe'.
}

\section{Brandon Malone and Elizabeth A. Campbell}

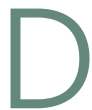
espite the reprieve from COVID-19 granted by vaccination programs, SARS-CoV-2 continues to ravage many communities worldwide. Vaccine shortages, public hesitancy and the emergence of new virus variants have hindered public health efforts to prevent the spread of COVID-19. Furthermore, SARS-CoV-2 is likely to become endemic ${ }^{1}$, leading to the emergence of vaccine-resistant variants and reinforcing the need to develop antiviral therapeutic agents. Molnupiravir (MK-4482, EIDD-2801) is a candidate antiviral that inhibits viral propagation through lethal mutagenesis by introducing errors in the viral genome. The biochemical and structural basis of how molnupiravir induces lethal mutagenesis has remained largely unexplored. Recently, Götte and colleagues reported biochemical results exploring the antiviral activity of molnupiravir and provided a compelling model to explain the mutagenic patterns observed in coronaviruses exposed to molnupiravir in cell culture ${ }^{2}$. In this issue of Nature Structural \& Molecular Biology, Cramer and colleagues ${ }^{3}$ further our understanding of this process by providing biochemical and structural data that reveal how molnupiravir introduces transition mutations into the SARS-CoV-2 genome. Together, the two studies offer complementary and comprehensive views of the mechanism of lethal mutagenesis and provide a platform for rational drug design.

Similar to other nucleoside analogs, molnupiravir targets the SARS-CoV-2 RNA-dependent RNA polymerase (RdRp), which mediates replication and transcription of the coronavirus genome. Viral RdRps are proven effective targets for inhibition, with several licensed nucleoside analogs that are used therapeutically ${ }^{1,4}$. Indeed, the only currently clinically approved antiviral used for the treatment of COVID-19 is remdesivir (Veklury), which targets the $\mathrm{RdRp}^{5}$. Similar to remdesivir, molnupiravir has been re-investigated as a coronavirus antiviral agent that leads to increased frequency of G-to-A and C-to- $\mathrm{U}$ transition mutations $^{6-9}$. Molnupiravir was also shown to inhibit propagation of the SARS-CoV, MERS-CoV and SARS-CoV-2 viruses, re-enforcing its pan-coronaviral inhibitory profile $^{8,10}$. Treatment with molnupiravir failed to induce viral-resistance mutations, which suggests a high genetic barrier to immune evasion ${ }^{6,7}$. Notably, in contrast to the antiviral nucleoside analogs fluorouracil (5-FU) and ribavarin ${ }^{11-13}$, molnupiravir is resistant to the proofreading exoribonuclease encoded by coronaviruses? which makes it an attractive target for further development. Thus, understanding the molecular basis of inhibition of molnupiravir reveals the lethal mutagenesis mechanism that will aid the informed design of related inhibitors.

Molnupiravir is a prodrug derivatized from the ribonucleoside analog $\beta$-D- $N^{4}$-hydroxycytidine (NHC) that is converted to its active form molnupiravir triphosphate (MTP) in the cell ${ }^{6}$. Both Gordon et al. ${ }^{2}$ and Kabinager et al. ${ }^{3}$ investigated the selective incorporation of MTP versus the natural nucleotides (ATP, GTP, UTP and CTP). They determined that MTP competes most effectively with CTP for incorporation into the product RNA. MTP also competes with UTP less efficiently, which suggests that MTP is unlikely to function as a UTP analog in the cellular milieu. After incorporation of molnupiravir (as the monophosphorylated MNP), RNA synthesis proceeds without stalling, which indicates that MNP does not act as a chain terminator, but is readily incorporated into the p-RNA. The lack of interruption to RNA synthesis may mitigate engagement of the proofreading complex, rendering it incapable of excising the incorporated MNP. Both groups hypothesized that the newly synthesized RNA transcript that contains MNP in lieu of cytidine could be subsequently used as the template RNA in the next round of viral RNA synthesis.

Kabinger et al. ${ }^{3}$ used solid-phase synthesis to generate a template RNA strand with an internal MNP base to specifically asses the role of an NHC as the templating base. By contrast, Gordon et al. ${ }^{2}$ enzymatically synthesized a template RNA containing MNP. Notably, they both observed that the templating MNP could form base pairs and subsequently incorporate either GTP or ATP into the new product-RNA strand. The finding that MNP can template ATP explains the previous observation of a G-to-A mutational bias after viral exposure to molnupiravir. The rationale for MNP recognizing both $\mathrm{G}$ and $\mathrm{A}$ is that the NHC base exists as two tautomers ${ }^{13}$. The hydroxylamine or amino form behaves chemically like $\mathrm{C}$ and enables base pairing with $G$, whereas the oxime or imino form mimics $U$ and pairs with A (Fig. 1f). Thus, tautomerization controls whether the correct (GTP) or incorrect (ATP) nucleotide is inserted and, therefore, whether mutagenesis will occur. By promoting incorporation of A instead of $\mathrm{G}$ into the nascrent RNA strand, the incorporated A templates a $U$ in the next round of RNA synthesis, resulting in an overall C-to-U error. A pathway for the C-to- $\mathrm{U}$ transition mutation that follows $\mathrm{C}^{+}$-to- $\mathrm{G}^{-}$to- $\mathrm{M}^{+}$-to-A-to- $\mathrm{U}^{+}$, in which the ' + ' and ' - ' refers to the positive- and negative sense strands, respectively, is shown in Fig. 1.This pathway supports the current evidence that there is an accumulation of $\mathrm{C}$-to- $\mathrm{U}$ mutations in the genomes of coronaviruses exposed to molnupiravir ${ }^{7,8}$.

In addition to the biochemical studies, Kabinger et al. ${ }^{3}$ determined high-resolution cryo-EM structures that show MNP templating either A or G in the RdRp active site, without notable perturbations of the RdRp active site or of the nucleic 
a

(+) SARS-CoV-2 genome

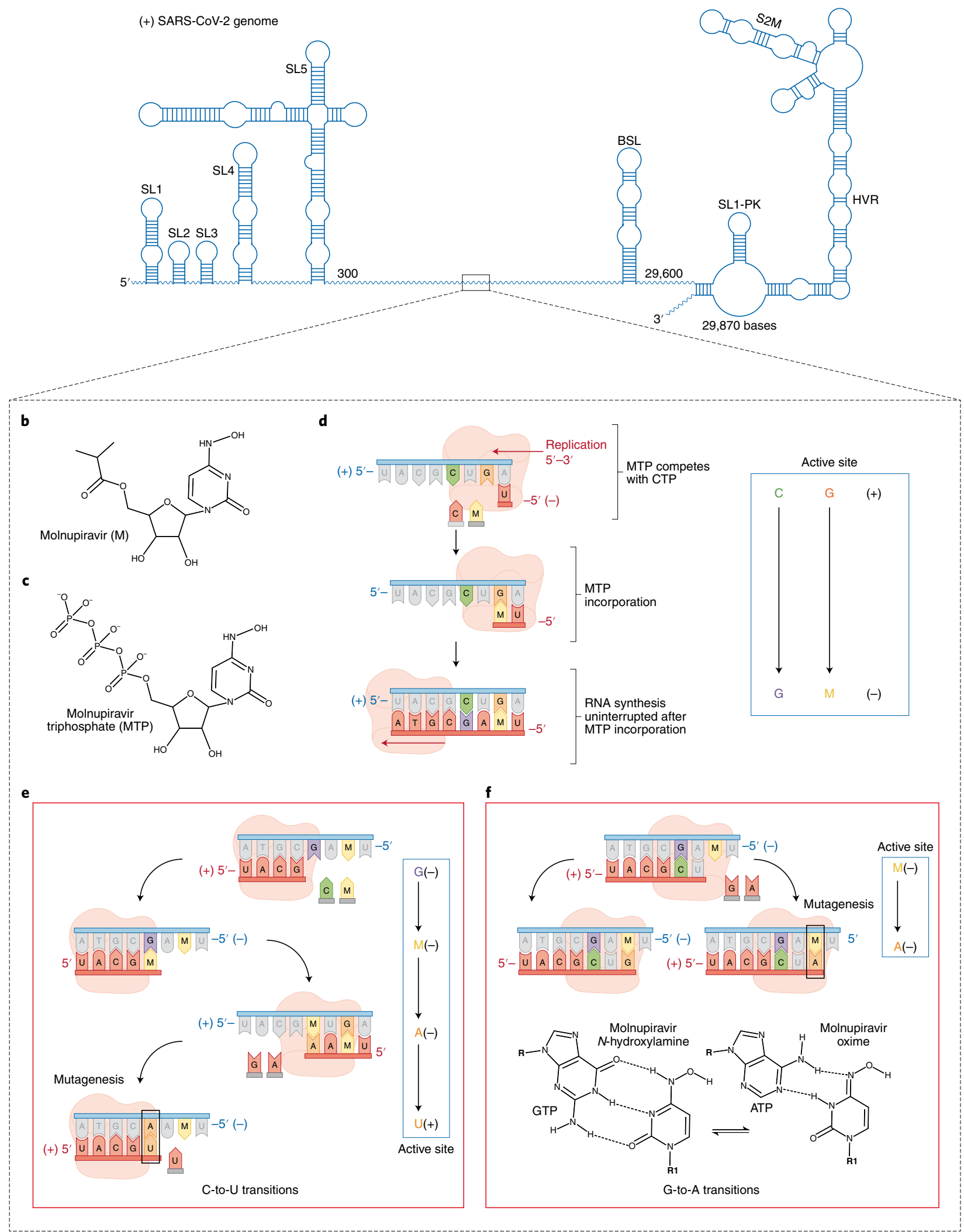

Fig. 1 | Schematization of mutagenic pathway by molnupiravir. a, Schematic representation of the SARS-CoV-2 genome. b,c, Chemical structure of molnupiravir (b) and the activated form, molnupiravir triphosphate (MTP) (c). d, MTP competes mainly with CTP for incorporation opposite G. e, Schematic depicting the $\mathrm{C}$-to- $U$ transition mutation pathway. The MNP-templated $\mathrm{A}$ incorporates a $\mathrm{U}$ in place of $\mathrm{C}$. $\mathbf{f}$, The template strand MNP base pairs either with $\mathrm{A}$ (incorrect nucleotide) or $\mathrm{G}$ (correct nucleotide), depending on the tautomer. 
acid scaffold. Although the cryo-EM maps cannot distinguish the two tautomeric forms, they reasonably assume that the amino form of molnupiravir base pairs with $\mathrm{G}$, forming three hydrogen bonds. By contrast, the imino tautomer forms two hydrogen bonds with A. Furthermore, the authors note that the hydrogen bond between $\mathrm{M}-\mathrm{A}$ and $\mathrm{M}-\mathrm{G}$ was suboptimal, possibly explaining its lower selectivity when compared with the natural NTPs. These structures thus provide a valuable direct visualization of how the NHC base of molnupiravir in the template strand base pairs with $\mathrm{G}$ and A to introduce G-to-A transition mutations.

Kabinger et al. ${ }^{3}$ and Gordon et al. ${ }^{2}$ therefore arrive at similar conclusions about the mechanism of lethal mutagenesis by molnupiravir, with subtle differences. Gordon et al. ${ }^{2}$ stress that the C-to-U transition mutations occur when MNP is incorporated in the template strand because the two tautomers of NHC exist more equally than its substrate triphosphate form. This model rationalizes the biochemical findings in the context of the high frequencies of G-to-A and C-to- $\mathrm{U}$ transition mutations in coronaviruses exposed to molnupiravir ${ }^{8}$. Another difference is the model provided by Kabinger et al. ${ }^{3}$ entertains A-to-G transitions, which can occur in coronaviruses exposed to molnupiravir, albeit at much lower frequencies ${ }^{8}$. Thus, although the biochemical evidence indicates that MTP is a better CTP analog, the genetic data suggest that MTP can compete weakly with UTP, inducing A-to-G transitions.

In summary, the two studies demonstrate that molnupiravir-induced lethal mutagenesis is minimally a two-step mechanism characterized by a relatively high selectivity of MTP for incorporation as a CTP analog and the indiscriminate incorporation of either ATP (mutagenesis) or GTP when MNP is localized in the templating strand. The erroneously incorporated AMP can subsequently template UTP incorporation, generating downstream C-to-U mutations. The accumulation of mutations pushes viral replication over the 'error threshold' that demarcates the replication fidelity required for viability. This mechanism distinguishes molnupiravir from remdesivir, which impedes the progression of viral RdRp, and provides insights into alternative mechanisms of RdRp inhibition. Finally, molnupiravir possesses excellent pharmacokinetic properties ${ }^{14}$, which include oral administration. An orally bioavailable antiviral will have far-reaching benefits in tackling the spread of COVID-19 in hard-to-reach communities worldwide. As with all therapeutic agents, off-target effects are a concern. In its triphosphate form, molnupirivar is a substrate for the mitochondrial RNA polymerase, which can also incorporate MTP as a U or C analog. Reassuringly, the same study noted that mitochondrial function over 14 days was not significantly inhibited ${ }^{15}$, and Sheahan et al. did not observe mutagenesis of host
$\mathrm{mRNA}^{8}$. However, it has been suggested that exposure to molnupiravir can be mutagenic to host DNA during host DNA replication ${ }^{16}$. Therefore, the potential off-target effects will require further investigation.

Brandon Malone and

Elizabeth A. Campbell ${ }^{凶}$

Laboratory of Molecular Biophysics, The Rockefeller

University, New York, NY, USA.

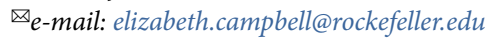

Published online: 13 September 2021

https://doi.org/10.1038/s41594-021-00657-8

References

1. Hall, M. D. et al. J. Infect. Dis. https://doi.org/10.1093/infdis/ jiab305 (2021).

2. Gordon, C. J., Tchesnokov, E. P., Schinazi, R. F. \& Götte, M. J. Biol. Chem. 297, 100770 (2021).

3. Kabinger, F. et al. Nat. Struct. Mol. Biol. https://doi.org/10.1038/ s41594-021-00651-0 (2021).

4. Vicenti, I., Zazzi, M. \& Saladini, F. Expert Opin. Ther. Pat. 31, 1-13 (2021).

5. Gordon, C. J. et al. J. Biol. Chem. 295, 6785-6797 (2020).

6. Yoon, J.-J. et al. Antimicrob. Agents Chemother. 62, e00766 (2018).

Agostini, M. L. et al. J. Virol. 93, e01348 (2019).

8. Sheahan, T. P. et al. Sci. Transl. Med. 12, eabb5883 (2020).

9. Urakova, N. et al. J. Virol. 92, e01965-17 (2018).

10. Wahl, A. et al. Nature 591, 451-457 (2021).

11. Bouvet, M. et al. Proc. Natl Acad. Sci. 109, 9372-9377 (2012).

12. Smith, E. C., Blanc, H., Vignuzzi, M. \& Denison, M. R. PLOS Pathog. 9, el003565 (2013).

13. Jena, N. R. Phys. Chem. Chem. Phys. PCCP 22,

28115-28122 (2020).

14. Toots, M. et al. Sci. Transl. Med. 11, eaax5866 (2019).

15. Sticher, Z. M. et al. Antimicrob. Agents Chemother. 64, e01719 (2020).

16. Zhou, S. et al. J. Infect. Dis. https://doi.org/10.1093/infdis/jiab2 (2021).

The authors declare no competing interests.

\title{
Is it a wrap? Nucleosome interactions of the BRCA1-binding partner, BARD1, steal the scene
}

\author{
Emerging findings provide compelling evidence that the BRCA1-binding partner BARD1 contributes yet further to \\ BRCA1 function. BARD1 is crucial for positioning the E2 ubiquitin-conjugating enzyme that confers specificity of \\ its ligase to residues on histone $\mathrm{H} 2 \mathrm{~A}$, and BARD1 also promotes DNA damage-induced chromatin recruitment \\ through an interaction with ubiquitin-conjugated Lys13 or Lys15 of H2A on the nucleosome core particle.
}

\section{Joanna R. Morris}

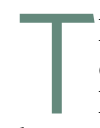
he BRCA1-BARD1 complex is a crucial tumor suppressor that is linked to the prevention of familial breast and ovarian cancers. The heterodimer has several proposed functions, including the regulation of DNA resection and the promotion of RAD51 loading through PALB2-BRCA2, which is required for homology-directed DNA repair. Despite the tremendous focus in the decades since the discovery of the BRCA1 gene, several critical questions have remained, if not completely unanswered, then with unsatisfactory solutions. Although a complete literature review is not possible here, some pertinent observations are that recruitment of BRCA1-BARD1 to damaged chromatin occurs through the 